\title{
Enhancement in resource allocation system for cloud environment using modified grey wolf technique
}

\author{
Soukaina Ouhame, Youssef Hadi \\ Faculty of Sciences and (FS), University Ibn Tofail Kenitra Morocco (UIT), Morroco
}

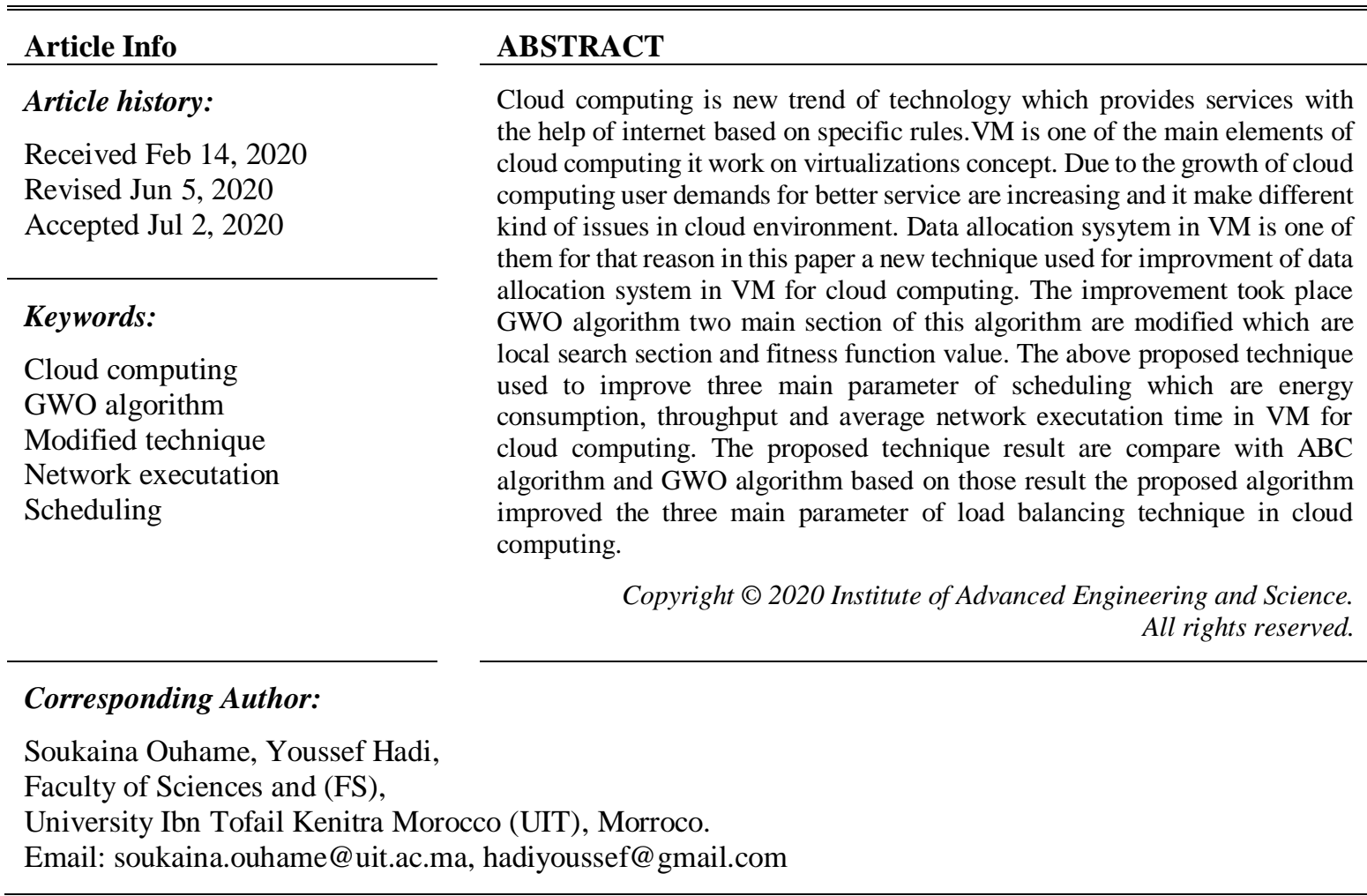

\section{INTRODUCTION}

Clouds computing becoming the most prominent technology due to it services because it provide the services with the help of internet and work on the base of pay and gain rule these services consist of hardware and software [1,2]. These applications are used in various field of life and improve the quality of our life. Cloud computing is emerging technology due to its main property which is know as virtualization process. Different physical machine run as virtual machine.Vitual machine play important role in cloud computing. Cloud computing become popular among the communities of business and researcher due to it virtualization property [3, 4]. Figure 1 show the working of cloud computing in which different devices are connected and get their required application. It is emerging field which is used for enormous-scale disseminated computing. Its framework used for ultimatum network admittance to achieve collective puddle of computing resource. As we know that cloud computing is increasing rapidly and user demand for better services in accurate and fast time [5, 6]. Cloud computing architecture consists of four layers. The inner layer is the hardware layer next to the inner layer is the infrastructure layer. Platform layer is above the infrastructure layer and the application layer is the outermost layer [7,8]. There are four types of cloud computing which are used in different field of life with specific rule and respective specification [9]. Types of cloud computing which private cloud, public cloud, hybrid cloud and community cloud computing. Virtualization is one of the main characteristic of cloud computing. Virtualization is the process in which physical instance of single application or resource share among multiple organization or users. This technique is done by assigning physical resources as logical form $[10,11]$. Its play an important role in cloud computing like sharing data as application but actually with 
the help of virtualization user share the infrastructure. Furthermore the main elements of virtualization are known as VM (virtual machine) [12, 13].

A single physical resource can appear as multiple resources this process can be achieved with the help of a virtual machine. It contains matched environment for a physical computer system its run an operating system and applications[14, 15]. It can be implemented through with the help of software, framework, and hardware. With the help of VM cloud computing reduce the real operating cost but still there are different issues facing by VM which are storage of resource, time, resource wastage, energy and data management $[16,17]$. Resource allocation is the process which provides service and storage for data or particular task. Resource allocation is one of the important issue still exit in cloud environment and it has various level of issue like scheduling, computational, performance, relocation response time, QoS, and energy management system $[18,19]$. In this study we focus on data allocation sysytem for VM system for cloud computing in the related work we study about the Grey Wolf technique which is used for scheduling process in VM and find out gap and desgin propsed technique for that gap.

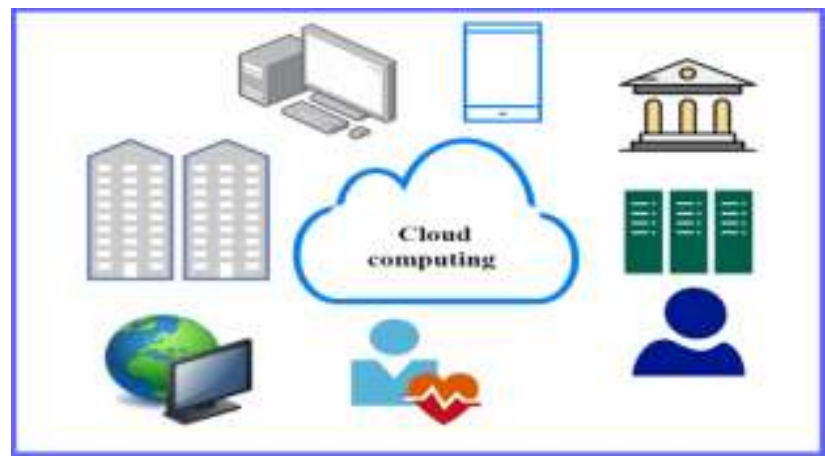

Figure 1. Cloud computing

\section{RESEARCH METHOD}

The author [20] proposed GWO-VMP algorithm for virtual machine placement for improvement in energy efficiency in cloud data centre. Because different active physical servers they are reducing the power consumption. Therefore the author used this technique to reduce the active host and make less energy consumption. The technique reduces the VM number and save more energy so the proposed technique is effective in energy management system for cloud computing.

The author [21] modified Grey Wolf technique for multi objective task scheduling in cloud computing. With the help of proposed technique improve the time, executation cost, and energy consumption. In the proposed technique the author change the fitness function and update additional term is incorporated using the combination of alpha and beta solutions due to these change it improve the result of the network.

The author [22] modified Grey Wolf technique for improvement in data scheduling in cloud computing. The modification took place in encircling and hunting operations have been modified by adding mean value to the equations. It is used for improving the efficiency of each wolf's motion and suitable path in searching area. With the help of this improvement the energy consumption and task executation improve in cloud computing.

The author [23] used Grey Wolf Optimization algorithm for parallel Job scheduling in cloud computing with the help of this technique it reduces the makespan and flow time. Changes are make in the fitness function and it makes quite significant improvment over the task executation in cloud computing.

The author [24] proposed a hybrid technique with the combination of particle swarm optimization (PSO), Biogeography-based optimization and Grey wolf technique used for resource allocation technique in cloud computing. The proposed technique improves the throughput using well balanced load across virtual machines and overcome the problem of trap into local optimum based on the result the proposed technique work more efficient.

The author [25, 26] used GWO algorithm for VM allocation system before the user sent request for resource manager which forward this message to the tuner it sent to all groups for resource but the researcher used the new technique which work on prioritization system therefore the load on server substantially reduced and improve response time and preparation time.

The author [26, 27] proposed Grey Wolf Algorithm it consist of different parameter which are alpha $(\alpha)$ wolf, beta $(\beta)$ wolf and delta $(\delta)$ wolf and the other reaming are called omega $(\omega)$ solutions. The author

Enhancement in resource allocation system for cloud environment using modified... (Soukaina Ouhame) 
modified first two step and improve the eliminate the waiting time in data allocation system of cloud computing. Based on result the proposed algorithm reduces the total query execution time and resource consumption. From the study of related work it seem that Grey Wolf algorithm used for data allocatin system for VM in cloud computing after the study of realted work it seen that Grey Wolf algorithm need some modification at some section for improvement in data allocation system in VM therefore in this study we improve two main section of the algorithm with the help of those changes we improve some of the main parameter of load balancing technique in cloud computing.

\section{METHODOLOGY}

The resource scheduling indicates the process of assigning the different task to different VM which is hosted on PMs of cloud data centre. Some situation these task are not properly distributed among different VM which affected the network performance. Therefore in this study proposed M.GWO technique which allocate the data to different VM in cloud computing. In the modified GWO technique two section are modified first section $\mathrm{ABC}$ algorithm searching section used for first iteration or local iteration because the GWO algorithm local search is not good as compare to ABC algorithm second modification make at fitness function of GWO technique it due to the improvement of energy system of the network. Cloud computing consist of different dynamic servers which provide the required of different user. Different VM assign different task by cloud data centre and they depends on different policies and as well as different load. If resource allocation not done properly in cloud computing it efficient on the entire network. Lets task [T1, T2.....Tn] and different VM are present as VM [VM1, VM2......VMn]. So in the proposed technique is design that we assign proper task to proper VM and for selection of VM randomly selection methord used by ABC local selection methord which express below.

$$
\frac{\mathrm{V}_{\mathrm{IJ}=\mathrm{X}_{\mathrm{ij}}+\mathrm{X}_{\mathrm{KJ}}}}{2}
$$

Where $\mathrm{K} \alpha[1,2 \ldots . \mathrm{n}]$ and $\mathrm{j} \alpha[1,2 \ldots \mathrm{n}]$ are randomly choice indexes Xij is the current food source with the ith employed bee is located.

$$
\mathrm{X}_{\mathrm{ij}+\theta}\left(\mathrm{X}_{\mathrm{ij}-} \mathrm{X}_{\mathrm{XJ}}\right)
$$

The Grey wolf optimizer technique consists of grouped in to 4 classes which are alpha, beta, delta and omega. Alpha present the leader from leader lowest domain present omega best solution is the top-level $\alpha$, the second-best solution is level $\beta$, the third-best solution is $\delta$ and other solutions belong to the lowest level $\omega$. From the second iteration the vectors $\vec{A}$ and $\vec{C}$ are calculated as.

$$
\begin{aligned}
& \vec{A}=2 \vec{a} \times \vec{r}-\vec{a} \\
& \vec{c}=2 \times \vec{r} 2
\end{aligned}
$$

Where $\vec{a}$ linearly decreases from 2 to 0 over the course of interaction and r1,r2 are random vector when start second iteration the following position update to the solutions.

$$
\begin{aligned}
& \overrightarrow{\mathrm{D}_{\sigma}}=\left[\overrightarrow{\mathrm{C}_{1}} * \overrightarrow{\mathrm{X}_{\sigma}}-\overrightarrow{\mathrm{X}}\right] \\
& \overrightarrow{\mathrm{D}_{\mathrm{B}}}=\left[\overrightarrow{\mathrm{C}_{1}} * \overrightarrow{\mathrm{X}_{\mathrm{B}}}-\overrightarrow{\mathrm{X}}\right] \\
& \overrightarrow{\mathrm{X}_{1}}=\overrightarrow{\mathrm{X}_{\alpha}}-\overrightarrow{\mathrm{A}_{1}} * \overrightarrow{\mathrm{D}_{\sigma}} \\
& \overrightarrow{\mathrm{X}_{3}}=\overrightarrow{\mathrm{X}_{\alpha}}-\overrightarrow{\mathrm{A}_{3}} * \overrightarrow{\mathrm{D}_{\sigma}} \\
& \overrightarrow{\mathrm{X}}(\mathrm{x}+1)=\left(\overrightarrow{\mathrm{X}_{1}}+\overrightarrow{\mathrm{X}_{2}}+\overrightarrow{\mathrm{X}_{3}}\right) / 3
\end{aligned}
$$

Where the length(i) is the length of cloudlet (i) and Pei is the number of processing element (PE) request by cloudlet (i) MIPSj (millions of instruction per second) PEi is the number of PE in VMj and $n$ is the number of VMs in a data center. Hence we need to choose the solution schedule such that the $\mathrm{F}$ value is minimized [28, 29]. 


$$
\begin{aligned}
& \mathrm{fi}=\sum_{\mathrm{i}=1}^{\mathrm{m}} \frac{\text { length } * \text { PEi }}{\text { MIP PEj }} \quad \text { for } \mathrm{j}=12 \ldots \mathrm{n} \mathrm{VMs} \\
& \mathrm{F}=\operatorname{Min}(\mathrm{fi})
\end{aligned}
$$

For time complexity of the proposed algorithm based scheduling depends of the number of VM (n) and the number of task are $(\mathrm{m})$ the group of VM taken $\mathrm{O}(\mathrm{n})$ and scheduling of task $\mathrm{O}(\mathrm{mn})$ and the over time complexity of proposed technique $=\mathrm{O}(\mathrm{mn})+\mathrm{O}(\mathrm{n})=\mathrm{O}(\mathrm{mn})$. Figure 2 show the flow chat for the proposed algorithm.

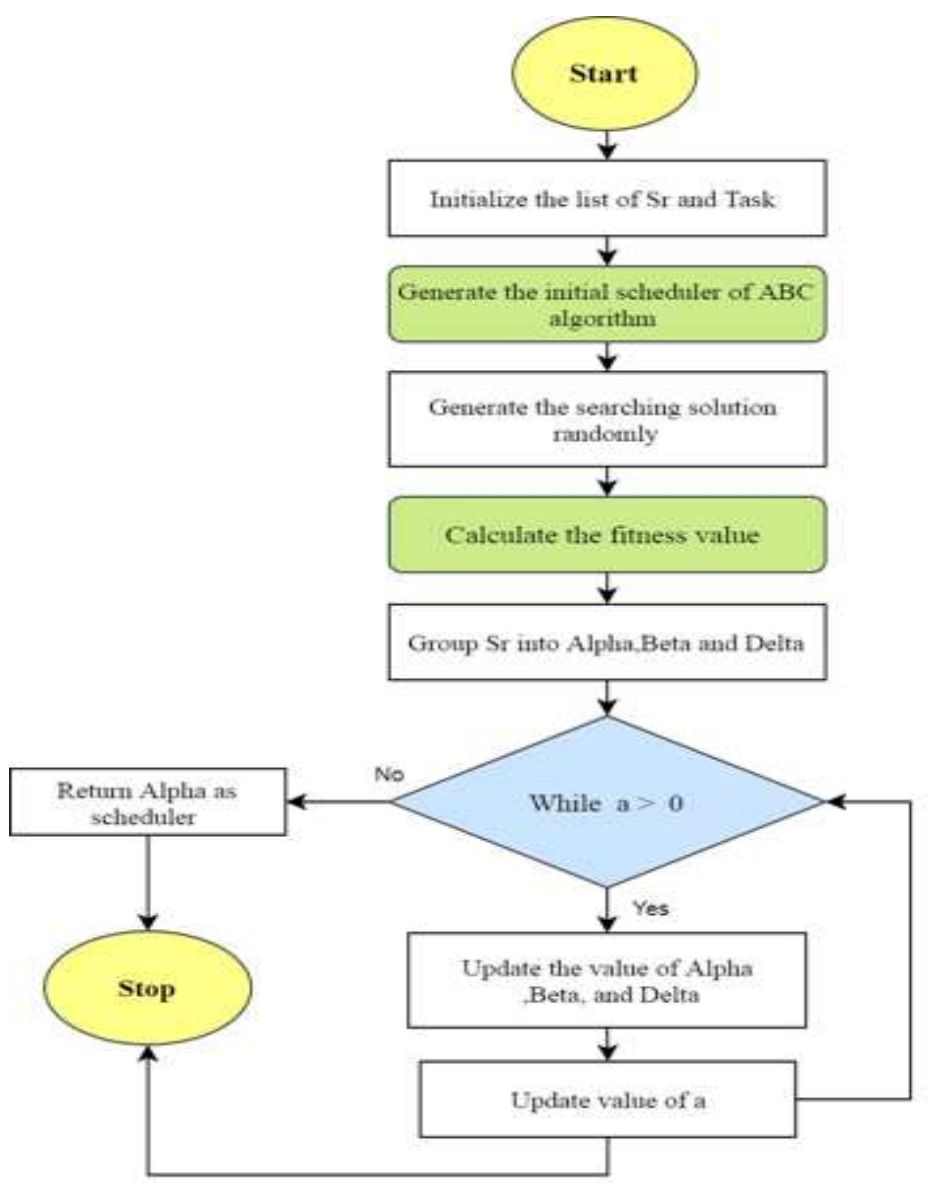

Figure 2. Flow chat of proposed technique

\section{Pseudocode of M.Grey Wolf Algorithm}

1. Initialization

2. Initialization the food source and evaluated the nectar amount (fitness) of food source.

3. Randomly generate the initial population of $\mathrm{N}$ gray wolves $\mathrm{Xi} i=1,2 \ldots \mathrm{N}$

4. Second iteration $\alpha \mathrm{A}$ and $\mathrm{C}$

5. Evaluate the fitness

6. Save the first three best solution $(\mathrm{X} \alpha, \mathrm{X} \beta, \mathrm{Xu})$

7. While $\mathrm{t}<$ matrix do>

8. For $\mathrm{i}=1 \mathrm{~N}$ do

9. Update the solution by using equation (3)

10. End for

11. Update $\alpha, A$ and $C$

12. Evaluate fitness of each individual update fitness of each individually update (Xo,X $\mathrm{X}, \mathrm{Xu})$

13. End while

14. Best solution retum

Figure 3. Pseudocode proposed technique 


\section{SIMULATION SETUP}

The proposed algorithm implemented using cloudsim 3.03 to check the three main parameter which are throughput, average network executation time and energy consumption of cloud computing. The proposed M.GWO algorithm result compare with $\mathrm{ABC}$ algorithm and GWO algorithm. After the simulation the result present in graphic form using origin software. Table 1 show the parameter which are used during simulation process. Table 1 shows the parameters according to this table the network has been established for simulation. Each simulation step two main parameter are change which are task number and user number to check the proposed technique performance.

Table 1. Experimental parameter used during simulation purpose

\begin{tabular}{ccc}
\hline Type & Parameter & Value \\
\hline Region & From 1 t0 5 & 5 \\
Data Centre & Number of data centre & 5 \\
& Number of hosts & $30 / 60$ \\
& Type of Manager & Time and space \\
& Bandwidth & 1000 \\
Virtual machine & Total number of VM & $5 * 4=20$ \\
& Number of processor per virtual machine & 4 \\
& Virtual machine memory & 512 \\
& Bandwidth in bit & 1000 \\
Task & VM image size & 1000 \\
& Number of task & $500 / 600$ \\
& Length of task & $150 \mathrm{~KB}$ \\
Memory & Type of manager & 100 \\
& Total memory & Time and space \\
& Number of processors & $204800 \mathrm{Mb}$ \\
& Total processor & 4 per VM \\
& Storage Memory & 120 \\
& Viable memory & $100000 \mathrm{Mb}$ \\
& & 10000 \\
\hline
\end{tabular}

\section{RESULT AND DISCUSSION}

This section presents the results which are taken from the simulation and compare with each other. Figure 4 show the throughput of 400 numberof tasks which are given to the network. Figure 4 compares the performance of throughput among all models. The number of VMs initialized as 20 number and 400 data or task given which are plated along $\mathrm{x}$-axis to check the throughput while receive throughput is plated in $\mathrm{y}$-axis. The proposed model completes more number of throughputs among all the modes. It means the proposed technique more efficient. Figure 5 show the throughput of 600 number of task in the network.

Figure 5 compares the performance of throughput among all models. The number of VMs initialized as 30 number and 600 data or task given which are plated along $\mathrm{x}$-axis to check the throughput while receive throughput is plated in y-axis. The proposed model completes more number of throughputs among all the modes. It means the proposed technique more efficient. Figure 6 show energy consumption of the network.

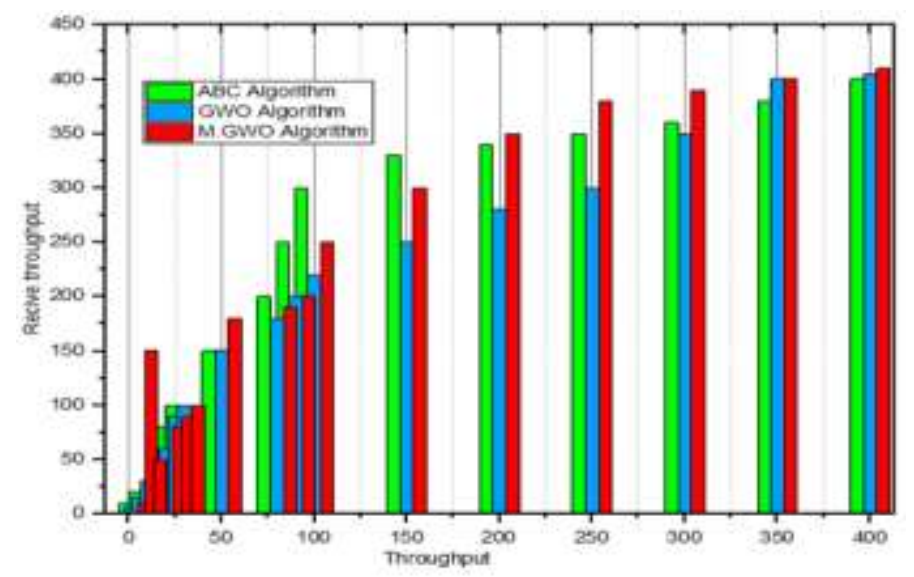

Figure 4: Throughput of 400 numbers of tasks 


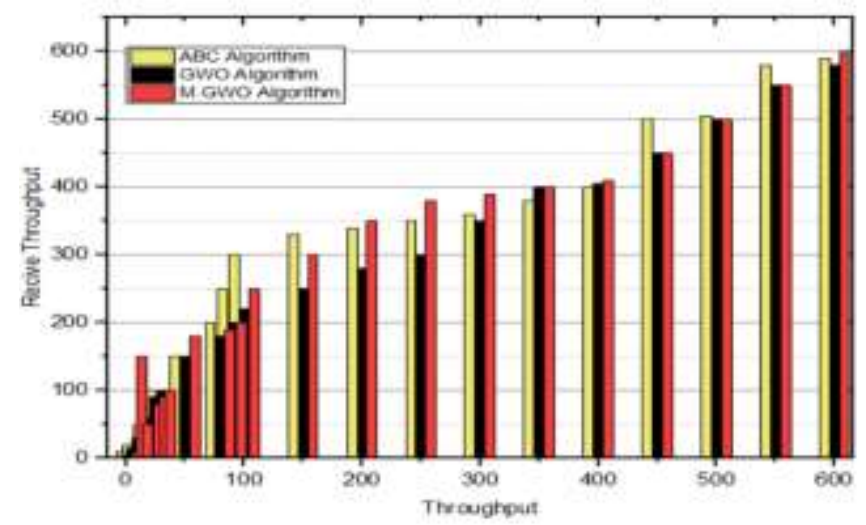

Figure 5. Throughput of 600 numbers of tasks
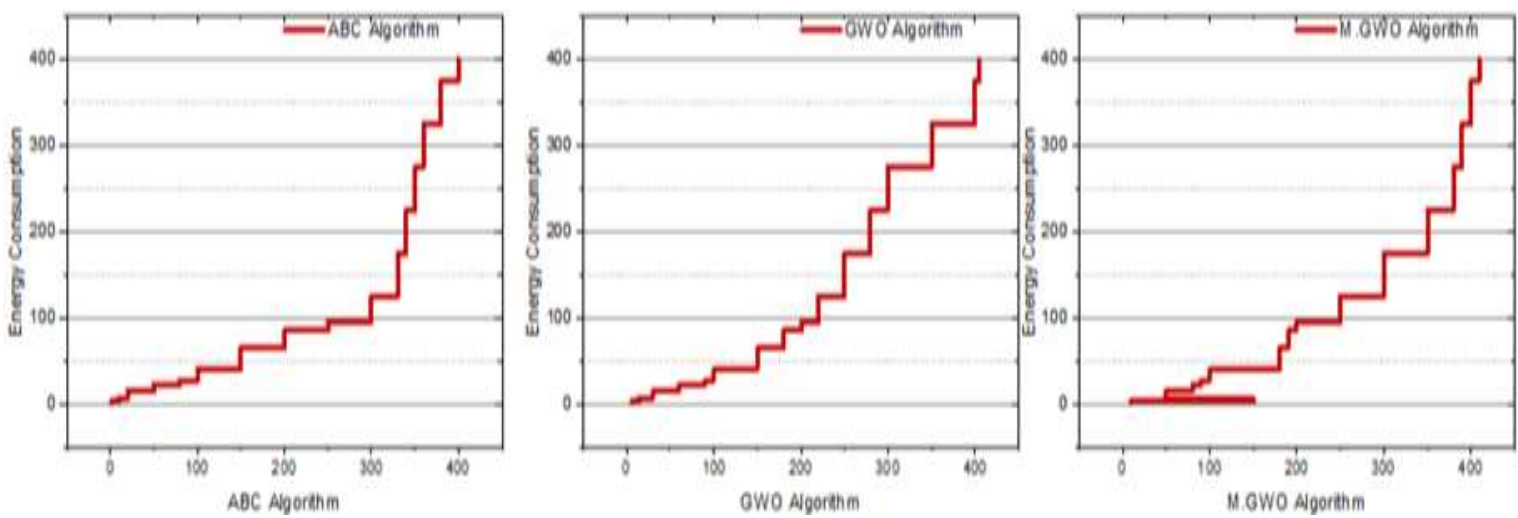

Figure 6. Energy consumption of network

Figure 6 show the energy consumption of the network based of the total time executation of the network the proposed technique take less amount of time of network it means the energy consumption is less then the other technique. Based on Figure 7 the average network executation time of network the proposed technique have less amount of time as compare the other technique.

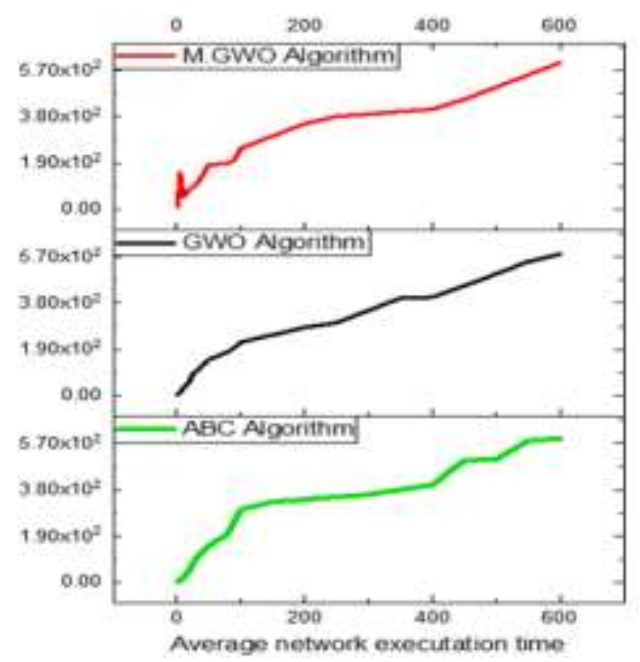

Figure 7. Average network executation time 


\section{CONCLUSION}

For the improvement of scheduling in cloud computing in this study a new technique design which is know as Modified-M.GWO. New technique design for improvement of three main parameter of scheduling which are throughput, energy consumption and average network executation time. Different number of task, different number of VM along with different number of user are used during executation of simulation. Based on those result the proposed technique is working more accurate in term of throughput, energy consumption and average network executation time in data allocation system of VM for cloud cpmuting. Based on the result the proposed algorithm improve the perfromance of VM data allocation sysytem of VMs in cloud data centre.

\section{REFERENCES}

[1] Aceto, G., Persico, V., \& Pescapé, A,’'Industry 4.0 and Health: Internet of Things, Big Data, and Cloud Computing for Healthcare 4.0," Journal of Industrial Information Integration, 100129, 2020.

[2] Badidi, E., Atif, Y., Sheng, Q. Z., \& Maheswaran, M. "On personalized cloud service provisioning for mobile users using adaptive and context-aware service composition." Computing, 101(4), pp. 291-318. 2019.

[3] Zhang, Q., Cheng, L., \& Boutaba, R. "Cloud computing: state-of-the-art and research challenges,” Journal of internet services and applications, 1(1), pp. 7-18, 2010.

[4] Ullah, A., Nawi, N. M., Uddin, J., Baseer, S., \& Rashed, A. H. "Artificial bee colony algorithm used for load balancing in cloud computing." 2019.

[5] Kamboj, P., Krishna, C. R., \& Reddy, S. R. N., "Real-Time Implementation of Scheduling Policies for Education Using Raspberry Pi: A Review." In Proceedings of 2nd International Conference on Communication, Computing and Networking, pp. 127-134, 2019. Springer, Singapore.

[6] Ullah, A., Umeriqbal, I. A. S., Rauf, A., Usman, O. Y., Ahmed, S., \& Najam, Z. An Energy-Efficient Task Scheduling using BAT Algorithm for Cloud Computing.

[7] Kumar, N. U., \& Siddappa, M., "Security for Virtualization in Cloud Services Using Duplication Method." Indonesian Journal of Electrical Engineering and Computer Science, Vol. 6, No. 2, 482-488: 2017.

[8] Mezgár, I., \& Rauschecker, U., "The challenge of networked enterprises for cloud computing interoperability". Computers in Industry, Vol. 65, No. 4, pp. 657-674, 2014.

[9] Mushtaq, M. F., Akram, U., Khan, I., Khan, S. N., Shahzad, A., \& Ullah, A., "Cloud computing environment and security challenges: A review." International Journal of Advanced Computer Science and Applications, 8(10), pp. 183-195, 2017.

[10] Espadas, J., Molina, A., Jiménez, G., Molina, M., Ramírez, R., \& Concha, D., “A tenant-based resource allocation model for scaling Software-as-a-Service applications over cloud computing infrastructures." Future Generation Computer Systems, 29(1), pp. 273-286, 2013.

[11] Mavridis, I., \& Karatza, H., "Combining containers and virtual machines to enhance isolation and extend functionality on cloud computing." Future Generation Computer Systems, 94, 6, 2019.

[12] Ullah, A., Nawi, N. M., Sutoyo, E., Shazad, A., Khan, S. N., \& Aamir, M., "Search Engine Optimization Algorithms for Page Ranking: Comparative Study.” International Journal of Integrated Engineering, Vol. 10, No. 6, 2018.

[13] Vemuri, H. K., Goyal, S., \& Awasthi, N., U.S. Patent No. 10,338,950. Washington, DC: U.S. Patent and Trademark Office, 2019.

[14] Umar, S., \& Baseer, S.,"Perception of cloud computing in universities of Peshawar," Pakistan. In 2016 Sixth International Conference on Innovative Computing Technology (INTECH) (pp. 87-91). IEEE, 2016

[15] Talebian, H., Gani, A., Sookhak, M., Abdelatif, A. A., Yousafzai, A., Vasilakos, A. V., \& Yu, F. R., "Optimizing virtual machine placement in IaaS data centers: taxonomy," review and open issues. Cluster Computing, 2019.

[16] Ullah, A., Nawi, N. M., Shahzad, A., Khan, S. N., \& Aamir, M., "An e-learning system in Malaysia based on green computing and energy level.” JOIV: International Journal on Informatics Visualization, 1(42), 2017.

[17] Zhou, Z., Liu, P., Feng, J., Zhang, Y., Mumtaz, S., \& Rodriguez, J., ”Computation resource allocation and task assignment optimization in vehicular fog computing: A contract-matching approach." IEEE Transactions on Vehicular Technology, 68(4), 3113-3125, 2019.

[18] Priya, V., Kumar, C. S., \& Kannan, R., "Resource scheduling algorithm with load balancing for cloud service provisioning." Applied Soft Computing, 76, pp. 416-424, 2019

[19] Hilman, M. H., Rodriguez, M. A., \& Buyya, R., "Multiple Workflows Scheduling in Multi-tenant Distributed Systems: Taxonomy and Future Directions.” ACM Computing Surveys (CSUR), 53(1), pp. 1-39, 2020.

[20] Al-Moalmi, A., Luo, J., Salah, A., \& Li, K., "Optimal virtual machine placement based on grey wolf optimization. Electronics," 8(3), 283, 2019

[21] Lingayat, A., Badre, R. R., \& Gupta, A. K., "Integration of linux containers in openstack: introspection, “' Indonesian Journal of Electrical Engineering and Computer Science, 12(3), pp. 1094-1105, 2018.

[22] Sreenu, K., \& Malempati, S., "MFGMTS: Epsilon constraint-based modified fractional grey wolf optimizer for multiobjective task scheduling in cloud computing," IETE Journal of Research, 65(2), 2019.

[23] Bouzary, H., \& Chen, F. F., "A hybrid grey wolf optimizer algorithm with evolutionary operators for optimal QoSaware service composition and optimal selection in cloud manufacturing," The International Journal of Advanced Manufacturing Technology, 101(9-12), 277, 2019 
[24] Kaur, S., Kaur, K., \& Chhabra, A., "Parallel job scheduling using grey wolf optimization algorithm for heterogeneous multi-cluster environment," Int J Comput Sci Eng, 5(10), 4, 2017.

[25] Sreenu, K., \& Malempati, S., "MFGMTS: Epsilon constraint-based modified fractional grey wolf optimizer for multiobjective task scheduling in cloud computing," IETE Journal of Research, 65(2), 2019.

[26] Meenakshi, A., Sirmathi, H., \& Ruth, J. A. (2019). Cloud computing-based resource provisioning using k-means clustering and GWO prioritization. Soft Computing, 23(21), 10781-10

[27] Naanaa, A., \& Belghith, S., "An efficient guided local search approach for multiuser detection in UWB systems. Physical Communication, 26, pp. 141-148, 2018.

[28] Liu, Z., Yang, B., Ma, C., Wang, S., \& Yang, Y., "Thermal error modeling of gear hobbing machine based on IGWOGRNN," The International Journal of Advanced Manufacturing Technology, 1, 2020.

[29] Baseer, S., \& Umar, S., "Role of cooperation in energy minimization in visual sensor network," In 2016 Sixth International Conference on Innovative Computing Technology (INTECH), pp. 447-452, 2016. 